\title{
Surgical Treatment of Ulcerative Colitis: A Review
}

\author{
Talha Sarigoz, MD*
}

Attending Surgeon, Develi-Hatice Muammer Kocaturk State Hospital, Turkey

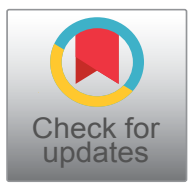

*Corresponding author: Talha Sarigoz, MD, Attending Surgeon, Develi-Hatice Muammer Kocaturk State Hospital, Seker Mahallesi, Molu Caddesi, Kayseri Sehir Hastanesi Genel Hastane Kat: 5 Kocasinan, Kayseri, 38000, Turkey, Tel: +90-542205-72-40

\begin{abstract}
Medical treatment which is focused on symptoms and reducing inflammation is the first step for the management of inflammatory bowel diseases [1]. Despite optimal medical therapy, up to $30 \%$ of the patients with ulcerative colitis will require surgery during their lifetime [2]. Colectomy and removal of rectum provide complete cure from the disease. But post-operative complications are major point of concern because most of the patients are worn out by extended medical therapy, malnutrition and hemorrhage $[3,4]$. Thereby, they are prone to post-operative morbidity and surgery decision is a delicate matter. This decision is best taken by an integrated, multidisciplinary approach between surgeon, gastroenterologist and patient. Patient status (e.g., nutritional status, previous treatment) and timing of surgery (e.g., urgent, elective) are the most important factors affecting surgical technique. The procedures may be divided into two categories based on timing; elective surgery and urgent surgery.
\end{abstract}

\section{Elective Surgery}

Reasons for elective surgery mostly rely on discontinuity of the medical therapy. Other reasons are dysplasia and cancer [5]. An elective surgery must be directed on removal of colon and rectum thus eliminating the target organ while achieving low mortality and morbidity rates and high quality of life. But with surgery comes complications, therefore different surgical techniques have been proposed to find better early and late outcomes and surgery for ulcerative colitis has evolved over the years (Table 1).

\section{Total proctocolectomy with ileostomy}

In both elective and emergency setting, till the late 1950 's, total proctocolectomy and ileostomy was the only procedure for ulcerative colitis patients [6]. But it is still a valid surgical option for selected patients who doesn't want pouch surgery and who are at risk for pouch failure such as elderly patients, patients with impaired sphincter function, additional comorbidities or history of anal surgery. Despite it is a one-stage procedure, it requires pelvic dissection and leaves patients with ostomy to live. Therefore complications are mostly stoma related like skin lesions, parastomal hernia, retraction and prolapse. Other complications are common to any procedure that includes pelvic dissection. Obstruction, infertility, sexual and bladder dysfunction and infection are of those. Reduced quality of life and functionality due to ostomy are considered pitfalls of this technique.

\section{Total proctocolectomy with ileal pouch anal anas- tomosis}

In 1980, Alan Parks and his friends described ileal pouch anal anastomosis (IPAA) and ever since most common approach for elective surgery has been restorative proctocolectomy with ileal pouch anal anastomosis [7]. Different pouch designs (e.g., S, J, W and H) were proposed but J pouch has been accepted widely because of functional results and quick construction [8]. Total proctocolectomy and IPAA can be performed in one, two and three stages depending on surgeon and patient condition [9]. One-stage procedure is mostly reserved for stable patients. For instance, patients who

Table 1: Indications for elective surgery.

- Refractoriness

- Complications due to medical therapy

- High risk of developing cancer or proven malignancy

\footnotetext{
Citation: Sarigoz T (2020) Surgical Treatment of Ulcerative Colitis: A Review. Int J Surg Res Pract 7:116. doi.org/10.23937/2378-3397/1410116 
Table 2: Complications following total proctocolectomy with ileal pouch anal anastomosis.

\begin{tabular}{|l|l|}
\hline Short Term & Long Term \\
\hline Wound infection & Pouchitis \\
\hline Pelvic abscess & Bowel obstruction \\
\hline Anastomotic stricture & Pouch dysfunction \\
\hline Bowel obstruction & Pouch fistula \\
\hline Pouch leak & Sexual dysfunction \\
\hline Bleeding & Reduction in fecundity \\
\hline
\end{tabular}

are young, healthy and those on no drugs are candidates for non-diverted surgery [2]. A two stage procedure which is the most common choice is composed of colectomy, pouch construction and loop ileostomy which is planned to be closed at a later time. A three stage procedure is composed of colectomy and ileostomy in the first stage, pouch construction in the second stage and ileostomy closure in the last stage. Multi-stage procedures are well suited for patients with poor nutritional status, acute colitis and those on high-dose steroid as they provide time for mucosal healing and detachment from immunosuppressives and steroids.

For IPAA, mortality rates have dropped below $1 \%$ in expert centers in the last decade and overall morbidity rates vary from $19 \%$ to $27 \%$ according to the literature [10]. Complications due to IPAA can be divided into two groups as short-term and long-term (Table 2). In an analysis of 3717 patients from Cleveland Clinic, shortterm complications were observed in $33 \%$ of patients and long-term complications in $29 \%$ [11].

The most important complication after IPAA is pelvic abscess which is a result of either anastomotic failure or infrequently, infected hematoma. Post-operative fever should arouse suspicion and urgent measures must be taken to reveal underlying reason. Because, pelvic sepsis may result in pouch failure, fistula and anastomotic stricture. Pouchogram with water-soluble contrast or pouchoscopy can be helpful to check integrity of the anastomosis [12]. If double stapled techniques are used, also the vagina must be examined for fistula. According to the size and extent of the pelvic abscess, there are different treatment approaches from broad-spectrum antibiotics to surgical intervention. For small abscess, antibiotics can be appropriate. For larger abscess, transanal drainage, drainage under radiological guidance are non-surgical measures but these methods may fail to control pelvic sepsis and eventually surgery is required [13]. For anastomotic leaks, diverting ileostomy is formed if not previously done. If total disruption of anastomosis is present, one should prefer externalizing the pouch.

The most common late-complication of IPAA is Pouchitis which presents itself as pelvic pain, cramps, fever and increased number of defecation. But there is lack of consensus regarding the definition of Pouchitis in the
Table 3: Indications for emergency surgery.

- Massive life-threatening hemorrhage

- Toxic megacolon

- Fulminant colitis who fail to respond medical therapy

- Perforation

literature and because of that reported rates of pouchitis whether early or late, differ from each other. For instance, in the study of Cleveland Clinic, Pouchitis rate following IPAA was $33 \%$. It was $48 \%$ at 10 years in the report of Mayo Clinic [14]. These values are high when compared to other reports $[15,16]$. Antibiotics mostly ciprofloxacin and metronidazole are the primary treatment and endoscopic biopsy is required sometimes for confirmation [17]. In rare cases of severe Pouchitis, ileostomy with or without pouch excision is required.

Sexual dysfunction and infertility are other concerns following pouch surgery. This is a result of pelvic dissection which is required for rectal resection. Although results of the published reports are controversial, mostly women are at risk and overall infertility rates vary from $38 \%$ to $64 \%[18,19]$. Before the operation, patients should be enlightened as to the possible negative effects on fertility.

In a systematic review it was stated that quality of life following IPAA improves a year later and UC patients become undistinguishable from the healthy ones irrespective of morbidity [20].

\section{Subtotal colectomy with ileorectal anastamosis}

During the 1960 's, subtotal colectomy with ileorectal anastomosis was proposed as an alternative method to total proctocolectomy and ileostomy. While lack of pelvic dissection and ileostomy construction are advantages of this technique theoretic risk of cancer arousing from the remnant rectal mucosa is a disadvantage. It requires follow up of the rectal mucosa for malignant change. Presence of rectal inflammation and loss of rectal compliance are contraindications to this technique. It is a surgical alternative in female fertile patients as it has no negative effect on fecundity [21].

\section{Urgent Surgery}

Main goal of the urgent surgery is retaining good health of the patient as soon as possible in order to perform future restorative procedures [22] (Table 3). Emergency surgery carries a great deal of mortality and morbidity when compared to elective surgery. In an analysis of ulcerative colitis patients that underwent emergency surgery, despite late medical advances, 30-day mortality rates were reported $13 \%$ [23]. In large series from Europe, following urgent surgery 30 -day mortality rates vary from $2.4 \%$ to $5.2 \%$ [24,25]. Higher rates should be expected in cases with advanced age, malnutrition and previous history of high dose immunosuppressive.

Subtotal colectomy with ileostomy and Hartmann's 
procedure is a safe and fast technique under emergency conditions. Distal stump may be either left in pelvis, created as a mucous fistula or placed superfascially. Also insertion of rectal drainage tube may decrease postoperative pelvic sepsis after this procedure [26]. After superfascial placement of distal colonic stump or creation of mucous fistula, fewer pelvic complications and less morbidity rates were reported [27]. But also there are reports that states intraperitoneal Hartmann's closure can be performed safely in selected patients.

Total colectomy is also an option but it requires pelvic dissection and high rates of hemorrhage, nerve damage and pelvic sepsis were reported. Regarding total proctocolectomy and IPAA, Heyvaert, et al. reported very high morbidity and anastomotic leak rate when compared to elective surgery and they specified it as a contraindication to emergency surgery [28].

\section{Minimally Invasive Surgery}

Minimally invasive surgery is the followed trend in the last decade. One of the first type laparoscopic surgeries is being used since 1992 for ulcerative colitis surgery [29]. With the technological development and growing experience, single instrument laparoscopic surgery and natural orifice transluminal endoscopic surgery have followed this trend [30]. Nowadays there are published reports using robotic systems for total proctocolectomy and IPAA. But they are mostly case reports and not enough to compare short-term and long-term outcomes [31,32].

Laparoscopic procedures minimize the surgical trauma to these patients. Thus they cause less pain and also improve cosmesis and relatively patient satisfaction. Despite demanding and time consuming, they provide patients with rapid recovery, comfort and reduced burden of adhesion related problems according to the literature [33]. There are reports comparing laparoscopy in both urgent and elective surgery. Yet, they are not sufficient to suggest it as a standard approach [34]. When technical expertise and facility are available, laparoscopy seems a better approach in elective setting.

\section{Age}

Total proctocolectomy and IPAA is now considered the standard treatment of ulcerative colitis requiring surgery according to latest guidelines but there are still controversies regarding IPAA can be performed safely in the elderly. Either elective or urgent, operative mortality rates have been found higher in older patients [35]. Age itself isn't a contraindication for IPAA but with advanced age comes comorbidities, muscular atrophy and sclerosis thereby anal sphincteric muscles weaken. Thus, careful planning must be put before the surgery. Total proctocolectomy and end-ileostomy is a better approach in elderly as it provides eradication of target organ and discharge of intestinal content easily when construction of pouch is contraindicated or not techni- cally possible. Subtotal colectomy and ileorectal anastomosis is another alternative in those patients. It is a quick operation with less dissection and avoiding stoma is an advantage. It also provides quick return to social life $[36,37]$.

\section{Hand-sewn versus Double-stapled Anastomosis}

In the original operation of IPAA, Parks denuded rectal mucosa to prevent recurrence and performed a hand-sewn anal anastomosis. Since the introduction of stapled techniques, there has been an ongoing debate over which technique is better. According to the large series comparing hand-sewn and double-stapled techniques, there wasn't significant difference regarding functional results and recurrence. In the recent studies, quality of life and functional outcomes after stapled-IPAA were significantly higher than hand-sewn-IPAA [38]. In the report of Kirat and Remzi, they compared both techniques in 3.109 patients and stated that patients undergoing stapled techniques had less complications and better quality of life [39].

\section{Acknowledgements}

\section{Funding}

None to declare.

\section{Conflict of interest}

None to declare.

\section{References}

1. Axel Dignass, James $O$ Lindsay, Andreas Sturm, Alastair Windsor, Jean-Frederic Colombel, et al. (2012) Second European evidence-based consensus on the diagnosis and management of ulcerative colitis part 2: Current management. J Crohns Colitis 6: 991-1030.

2. Howard Ross, Scott R Steele, Mika Varma, Sharon Dykes, Robert Cima, et al. (2014) Practice parameters for the surgical treatment of ulcerative colitis. Dis Colon Rectum 57: 5-22.

3. Wojciech Blonski, Anna M Buchner, Gary R Lichtenstein (2014) Treatment of ulcerative colitis. Curr Opin Gastroenterol 30: 84-96.

4. Bordeianou L, Maguire L (2013) State-of-the-art surgical approaches to the treatment of medically refractory ulcerative colitis. J Gastrointest Surg 17: 2013-2019.

5. Andersson P, Soderholm JD (2009) Surgery in ulcerative colitis: Indication and timing. Dig Dis 27: 335-340.

6. Scoglio D, Ahmed AU, Fichera A (2014) Surgical treatment of ulcerative colitis: lleorectal vs ileal pouch-anal anastomosis. World J Gastroenterol 20: 13211-13218.

7. Parks AG, Nicholls RJ, Belliveau P (1980) Proctocolectomy with ileal reservoir and anal anastomosis. Br J Surg 67: 533-538.

8. Keighley MR, Yoshioka K, Kmiot W (1988) Prospective randomized trial to compare the stapled double lumen pouch and the sutured quadruple pouch for restorative proctocolectomy. Br J Surg 75: 1008-1011.

9. Grucela A, Steinhagen RM (2009) Current surgical management of ulcerative colitis. Mt Sinai J Med 76: 606-612. 
10. Charles N Bernstein, Siew C Ng, Peter L Lakatos, Bjorn Moum, Edward V Loftus Jr, et al. (2013) A review of mortality and surgery in ulcerative colitis: Milestones of the seriousness of the disease. Inflamm Bowel Dis 19: 2001-2010.

11. Victor Warren Fazio, Ravi P Kiran, Feza H Remzi, John Calvin Coffey, Helen Mary Heneghan, et al. (2013) Ilea pouch anal anastomosis: Analysis of outcome and quality of life in 3707 patients. Ann Surg 257: 679-685.

12. Kirat HT, Remzi FH (2010) Technical aspects of ileoanal pouch surgery in patients with ulcerative colitis. Clin Colon Rectal Surg 23: 239-247.

13. Bach SP, Mortensen NJ (2007) Ileal pouch surgery for ulcerative colitis. World J Gastroenterol 13: 3288-3300.

14. Dieter Hahnloser, John H Pemberton, Bruce G Wolff, Dirk R Larson, Brian S Crownhart, et al. (2004) The effect of ageing on function and quality of life in ileal pouch patients: A single cohort experience of 409 patients with chronic ulcerative colitis. Ann Surg 240: 615-621.

15. Peter WM, Patricia LR, David JS (1993) Long-term results of the ileoanal pouch procedure. Arch Surg 128: 500-503.

16. Rickard MJFX, Young CJ, Bissett IP, Stitz R, Solomon MJ, et al. (2007) Ileal pouch-anal anastomosis: The Australasian experience. Colorectal Dis 9: 139-145.

17. Shen B, Achkar JP, Lashner BA, Ormsby AH, Remzi FH, et al. (2001) Endoscopic and histologic evaluation together with symptom assessment are required to diagnose pouchitis. Gastroenterology 121: 261-267.

18. Julie AC, Emile Tan, Julian Teare, Teoh GT, Raj Rai, et al. (2007) The effect of restorative proctocolectomy on sexual function, urinary function, fertility, pregnancy and delivery: A systematic review. Dis Colon Rectum 50: 1128-1138.

19. Kasper Ording Olsen, Svend Juul, Ina Berndtsson, Tom Oresland, Søren Laurberg, et al. (2002) Ulcerative colitis: Female fecundity before diagnosis, during disease, and after surgery compared with a population sample. Gastroenterology 122: 15-19.

20. Patrick BM, Zaid Khot, Kelly NV, Michael Ott, Luc Dubois (2015) Quality of life after total proctocolectomy with ileostomy or ipaa: A systematic review. Dis Colon Rectum 58: 899-908.

21. Pierre-Eugène Mortier, Luc Gambiez, Medhi Karoui, Antoine Cortot, Jean-Claude Paris, et al. (2006) Colectomy with ileorectal anastomosis preserves female fertility in ulcerative colitis. Gastroenterol Clin Biol 30: 594-597.

22. Nicholls RJ (2002) Review article: Ulcerative colitis-surgical indications and treatment. Aliment Pharmacol Ther 16: 2528.

23. Supriya S Patel, Madhukar S Patel, Melanie Goldfarb, Adrian Ortega, Glenn T Ault, et al. (2013) Elective versus emergency surgery for ulcerative colitis: A National Surgical Quality Improvement Program analysis. Am J Surg 205: 333-337.

24. Omar Faiz, Janindra Warusavitarne, Alex Bottle, Paris $P$ Tekkis, Sue K Clark, et al. (2010) Nonelective excisional colorectal surgery in English National Health Service Trusts: A study of outcomes from hospital episode statistics data between 1996 and 2007. J Am Coll Surg 210: 390401.
25. Anders Tøttrup , Rune Erichsen, Claus Sværke, Søren Laurberg, Henrik Toft Srensen (2012) Thirty-day mortality after elective and emergency total colectomy in danish patients with inflammatory bowel disease: A population-based nationwide cohort study. BMJ Open 2.

26. Karch LA, Bauer JJ, Gorfine SR, Gelernt IM (1995) Subtotal colectomy with hartmann's pouch for inflammatory bowel disease. Dis Colon Rectum 38: 635-639.

27. Carter FM, McLeod RS, Cohen Z (1991) Subtotal colectomy for ulcerative colitis: Complications related to the rectal remnant. Dis Colon Rectum 34: 1005-1009.

28. Heyvaert G, Penninckx F, Filez L, Aerts R, Kerremans R, et al. (1994) Restorative proctocolectomy in elective and emergency cases of ulcerative colitis. Int $\mathrm{J}$ Colorectal Dis 9: 73-76.

29. Peters WR (1992) Laparoscopic total proctocolectomy with creation of ileostomy for ulcerative colitis: Report of two cases. J Laparoendosc Surg 2: 175-178.

30. Holder-Murray J, Marsicovetere P, Holubar SD (2015) Minimally invasive surgery for inflammatory bowel disease. Inflamm Bowel Dis 21: 1443-1458.

31. Rodrigo Pedraza, Chirag B Patel, Diego I Ramos-Valadez, Eric M Haas (2011) Robotic-assisted laparoscopic surgery for restorative proctocolectomy with ileal $\mathrm{J}$ pouch-anal anastomosis. Minim Invasive Ther Allied Technol 20: 234239.

32. Aaron $T$ Miller, Julia R Berian, Michele Rubin, Roger $D$ Hurst, Alessandro Fichera, et al. (2012) Robotic-assisted proctectomy for inflammatory bowel disease: A casematched comparison of laparoscopic and robotic technique. J Gastrointest Surg 16: 587-594.

33. Xiao-Jian Wu, Xiao-Sheng He, Xu-Yu Zhou, Jia Ke, Ping Lan (2010) The role of laparoscopic surgery for ulcerative colitis: Systematic review with meta-analysis. Int J Colorectal Dis 25: 949-957.

34. Fowkes L, Krishna K, Menon A, Greenslade GL, Dixon AR (2008) Laparoscopic emergency and elective surgery for ulcerative colitis. Colorectal Dis 10: 373-378.

35. Hiroki Ikeuchi, Motoi Uchino, Hiroki Matsuoka, Toshihiro Bando, Akihiro Hirata, et al. (2014) Prognosis following emergency surgery for ulcerative colitis in elderly patients. Surg Today 44: 39-43.

36. Pastore RL, Wolff BG, Hodge D (1997) Total abdominal colectomy and ileorectal anastomosis for inflammatory bowel disease. Dis Colon Rectum 40: 1455-1464.

37. Saito $Y(1995)$ Total colectomy and ileorectal anastomosis in ulcerative colitis. J Gastroenterol 8: 131-134.

38. Richard E Lovegrove, Vasilis A Constantinides, Alexander G Heriot, Thanos Athanasiou, Ara Darzi, et al. (2006) A comparison of hand-sewn versus stapled ileal pouch anal anastomosis (IPAA) following proctocolectomy: A meta-analysis of 4183 patients. Ann Surg 244: 18-26.

39. Hasan T Kirat, Feza H Remzi, Ravi P Kiran, Victor W Fazio (2009) Comparison of outcomes after hand-sewn versus stapled ileal pouch-anal anastomosis in 3,109 patients. Surgery 146: 723-730.

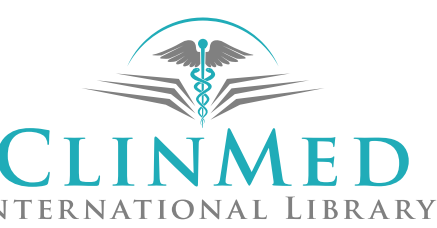

